\title{
Digital inertial algorithm for recording track geometry on commercial shinkansen trains
}

\author{
M. Kobayashi, Y. Naganuma, M. Nakagawa \& T. Okumura \\ Technology Research and Development Department, \\ Central Japan Railway Company, Japan
}

\begin{abstract}
The inertial method is based on a simple law that states that the double integration of acceleration gives its displacement, and is one of the general methods used for recording track geometry. In the inertial method, a high-pass filter (HPF) is essential to ensure stable calculation of the double integration of acceleration. The previous analog inertial measuring system remained wave distortion because the system used analog HPFs, which had a non-linear phase property. To avoid wave distortion caused by analog HPFs, a new algorithm was developed for digital inertial measurement that utilizes the measurement property of the $10 \mathrm{~m}$ versine. A new track geometry recording device using this algorithm was produced, capable of being installed on commercial shinkansen trains. This device, called RAIDARSS 3, measures $10 \mathrm{~m}$ versine track irregularities from double integration of the axle box acceleration in real time. If the measured track irregularities exceed the predetermined target maintenance values, the measured values and locations of the irregularities are automatically reported to the train control center and track maintenance depots, and slow speed operation is directed, if necessary. To confirm the measurement precision of this device, an experimental prototype was tested on a shinkansen test train. Results showed that the output of the prototype achieved good correlation with data from the existing track geometry car. The new RAIDARSS 3 track condition monitoring device will be installed on six N700 train sets during fiscal year 2008.
\end{abstract}

Keywords: inertial method, recording track geometry, $10 \mathrm{~m}$ versine. 


\section{Introduction}

For recording track geometry, high-precision measurement is required to ensure safe train operation and improvement of riding comfort. Moreover, since highspeed and high-density train lines require a high level of safety, a higher frequency of track geometry recording is required to more quickly identify extraordinary conditions. For recording track geometry, generally a differential method such as the $10 \mathrm{~m}$ versine and inertial method are utilized. The differential method enables track geometry to be measured independent of the speed, but requires more than three measurement points and a special car with special bogies to attach the measuring devices.

On the Tokaido shinkansen line, a track-inspection train called Doctor Yellow runs once every 10 days to monitor conditions of facilities on the ground (Fig. 1). A track geometry car on Doctor Yellow that has special bogies with attached measuring devices measures track irregularities using the differential method at a speed of $270 \mathrm{~km} / \mathrm{h}$, equal to that of commercial trains (Figs. 2, 3).

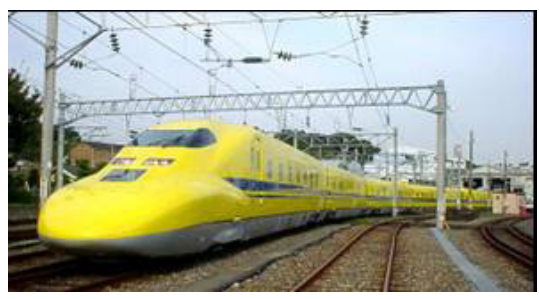

Figure 1: Track-inspection car "Doctor Yellow".

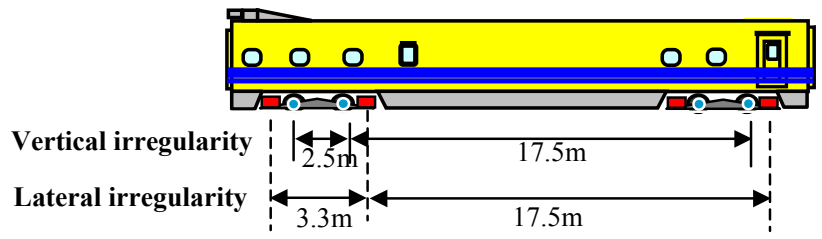

Figure 2: $\quad$ Track geometry car on Doctor Yellow (differential method).

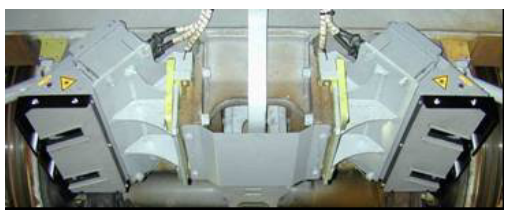

(a) Measuring device for lateral irregularity

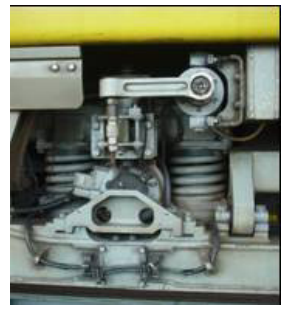

(b) Measuring device for vertical irregularity

Figure 3: $\quad$ Track measuring devices (differential method). 


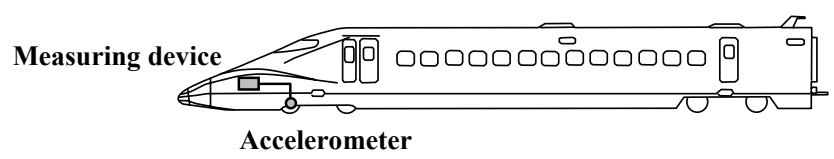

Figure 4: $\quad$ Measuring system (inertial method).

The measured track irregularities are then converted to $10 \mathrm{~m}$ versine values, which identify spots on tracks that require immediate maintenance. On the other hand, the inertial method enables track geometry to be measured from the acceleration at one point. Therefore, this system has the advantages of being simpler and not requiring a special car (Fig. 4). However, the disadvantage of this method is that at low speeds the acceleration signal becomes very small, and thus not able to maintain measurement precision in areas where trains are traveling slowly, such as near stations. On the Tokaido shinkansen line, an inertial measuring device called TRASC [1] (TRAck State Confirming machine) installed on a track state confirming car is used to confirm safety before trains are put into service. Moreover, an inertial measuring device called TRIPS (TRack Information Processing System) [2] installed on a high-speed test train named " $300 \mathrm{X}$ " is used to measure track irregularities at speeds more than 400 $\mathrm{km} / \mathrm{h}$ to analyze the relationship between car behavior and track irregularities. However, the outputs of these devices remained wave distortion because the system used an analog HPF. Due to the HPF's non-linear phase property, sufficient measurement precision has not been achieved. Therefore, to realize an inertial measuring device using digital signal processing, which does not cause wave distortion, an algorithm specifically for the new device was developed.

\section{Problems with previous inertial measuring devices}

The inertial measuring device outputs the displacement through double integration of the acceleration. If an acceleration signal has a slight offset, the output of the integrator soon becomes saturated because the gain of the integrator at $0 \mathrm{~Hz}$ approaches infinity. To avoid saturation, a HPF is added before the integrator. The HPF must be a spatial filter whose cut-off frequency varies with vehicle speed in order to maintain the desired bandwidth of track irregularity. Since the above spatial HPF was realized only using an analog circuit, it was not possible to achieve the linear phase property. This, then, resulted in an output signal with unavoidable wave distortion. Additionally, measured waveforms of two reverse directions are differ greatly, especially around the cut-off wavelength. Fig. 5 shows an example of a waveform from TRIPS. For these reasons, output data from previous inertial measuring devices could not be widely utilized.

Utilizing a digital phase compensation filter was proposed as one way to correct the wave distortion [3]. Since waveform distortion is clearly due to the characteristics of the analog high-pass filter, the distortion can be corrected by reversing the phase of the output signal. This is done by passing the output of the 


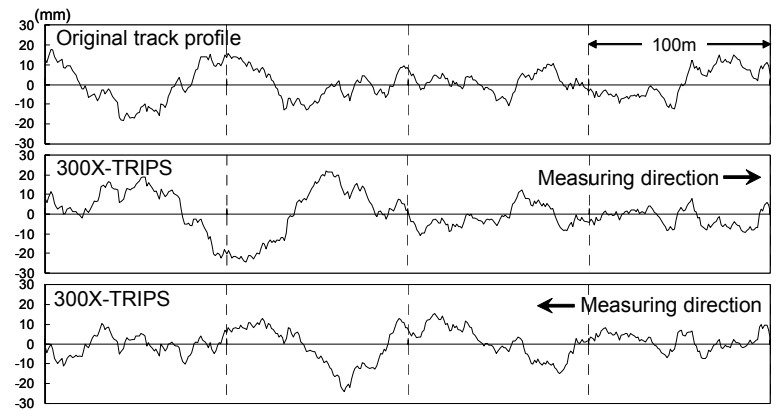

Figure 5: $\quad$ Waveform distortion of analog inertial system (300X-TRIPS).

analog inertial measuring device through a digital phase compensation filter. Another way to avoid wave distortion is to send the digital signal through an integrator and HPF, because the digital filter can easily realize the linear phase property. However, adopting digital signal processing to the integration generates other difficulties that must be solved.

\section{Algorithms for the digital inertial measuring device}

\subsection{Algorithm using digital HPFs}

First, an algorithm that uses a digital HPF before the integrator is examined. Fig. 6 shows a block diagram of this method. Track irregularity calculated through this circuit in the time domain is resampled to the distance and output for the value of $10 \mathrm{~m}$ versine.

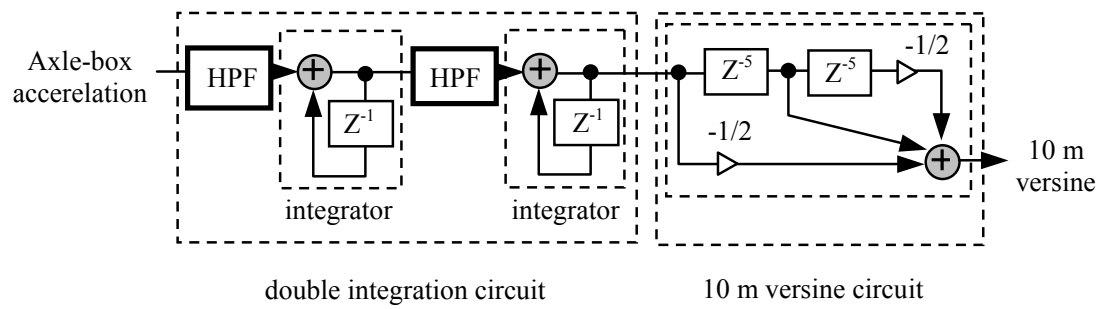

Figure 6: $\quad$ Block diagram of digital integration using digital HPFs.

For a sampling distance of $1 \mathrm{~m}$, the $10 \mathrm{~m}$ versine method is expressed by Equation (1):

where,

$$
y(\xi)=x(\xi)-\frac{x(\xi-5)+x(\xi+5)}{2}
$$

$y(\xi)$ : measured $10 \mathrm{~m}$ versine signal 
$x(\xi)$ : original track profile on the ground

The digital inertial measuring device has the following required measuring conditions.

- Maximum measuring speed is $300 \mathrm{~km} / \mathrm{h}$

- Minimum wavelength included in track irregularity is $3 \mathrm{~m}$.

- Sampling distance is $0.25 \mathrm{~m}$, which is equal to the track geometry car.

Under these conditions, the HPF must have a very low cutoff frequency to cover low speeds and long wavelengths. If a general digital FIR (finite impulse response) filter is used as the HPF, many filter coefficients would be required. Sum-product calculations for several thousand points of the filter coefficients would be a very heavy processing load even for today's high-speed processors. Therefore, to satisfy the performance of the DSP used, the digital HPF in this method is designed so the filter coefficients have less than 4001 points. As a result, the cutoff frequency for the designed HPF is $0.5 \mathrm{~Hz}$ for $600 \mathrm{~Hz}$ sampling data. This frequency is equivalent to a wavelength of $50 \mathrm{~m}$ at $90 \mathrm{~km} / \mathrm{h}$. Thus, when track irregularity is required to measure up to a wavelength of $50 \mathrm{~m}$, the desired wavelength band cannot be achieved at speeds less than $90 \mathrm{~km} / \mathrm{h}$.

\subsection{Algorithm using frequency variable difference filter}

From Equation 1, a transfer function for a $10 \mathrm{~m}$ versine measurement on the $\mathrm{z}$ plane yields Equation (2). However, in this equation, the sampling distance is 1.0 $\mathrm{m}$ with an output delay of $5 \mathrm{~m}$ to satisfy the law of causality.

$$
H_{10}(z)=-\frac{1}{2}+z^{-5}-\frac{1}{2} z^{-10} .
$$

Furthermore,

$$
H_{10}(z)=-\frac{1}{2}\left(1-2 z^{-5}+z^{-10}\right)=-\frac{1}{2}\left(1-z^{-5}\right)^{2} .
$$

Equation (3) shows that a characteristic of the $10 \mathrm{~m}$ versine consists of two difference filters and one multiplier. Fig. 7 shows a block diagram of the $10 \mathrm{~m}$ versine method, and Fig. 8 shows the characteristics of a finite difference filter. As shown in Fig. 8, because the difference filter has a high-pass characteristic in the divided $10 \mathrm{~m}$ versine method, and the DC gain is zero $(\mathrm{f}=0 \mathrm{~Hz})$, this filter can utilize integration stabilization [4]. Since the difference filter operates by taking the difference between the input signal and its delayed signal, the processing load is very light. Furthermore, a variable frequency filter can easily be made by changing the delay corresponding to the vehicle speed.

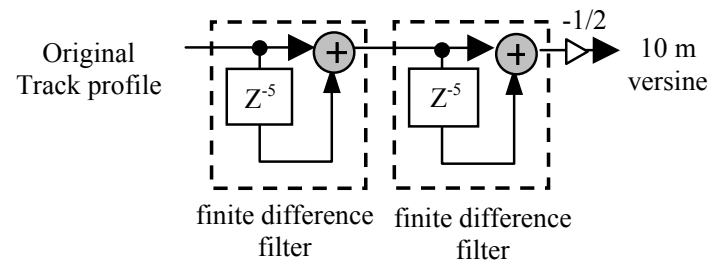

Figure 7: Block diagram of $10 \mathrm{~m}$ versine method. 


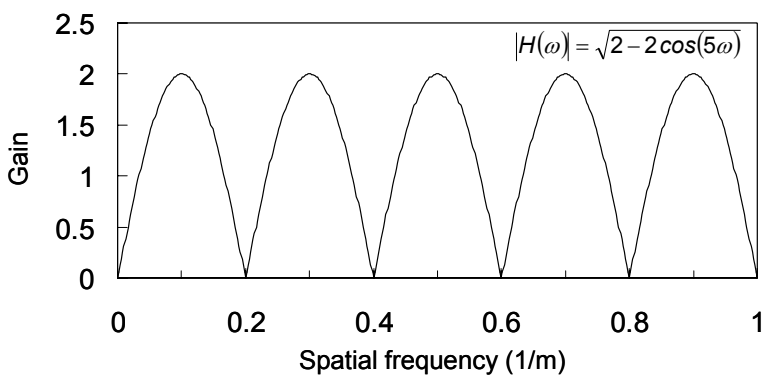

Figure 8: Frequency response of difference filter.

With a sampling frequency of 1.0 second, the characteristics of the $10 \mathrm{~m}$ versine method in the time domain yields Equation 4.

$$
H_{10}(z)=-\frac{1}{2}\left(1-z^{-L}\right)^{2} \text {. }
$$

Where $L$ is as follows and $v$ is the vehicle speed.

$$
L=\frac{5}{v} \text {. }
$$

Equation 5 is the transfer function for the simplest digital integrator on the zplane.

$$
H_{I}(z)=\frac{1}{1-z^{-1}} .
$$

Using Equations 4 and 5, the integral and versine composite calculation is expressed as Equation 6.

$$
H_{F D}(z)=H_{10} \cdot H_{I}=-\frac{1}{2}\left(\frac{1-z^{-L}}{1-z^{-1}}\right)^{2} .
$$

In this paper, this digital inertial measurement algorithm is called "Frequency variable difference filter". Fig. 9 shows a block diagram of this algorithm.

Since this system is mainly composed of adders, and uses only one multiplier, the hardware load is very light. Moreover, this algorithm enables much lower measurable lower limit speed.

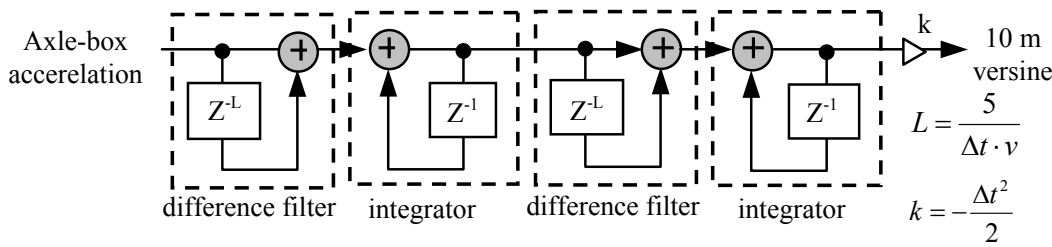

Figure 9: Block diagram of Frequency variable difference filter. 


\section{Applying digital inertial measuring devices on commercial Tokaido shinkansen trains}

On the Tokaido shinkansen line, in addition to track geometry recording every 10 days, the car body acceleration of commercial trains has been measured every day since the inauguration of service in 1964. Today, car body acceleration is automatically measured several times a day using a device called RAIDARSS (Real-time Acceleration Inspection Device with Automatic Recording System for Shinkansen), which is currently installed on four 700-series shinkansen train sets. Fig. 10 shows an outline of RAIDARSS $2+$. If the measured accelerations exceed the predetermined target maintenance values, RAIDARSS 2+ automatically reports the measured values and locations of the irregularities to the train control center and track maintenance depots via the train radio. Depending on the acceleration value, slow speed operation is engaged to ensure safe train operation. Table 1 shows the main functions of RAIDARSS $2+$.

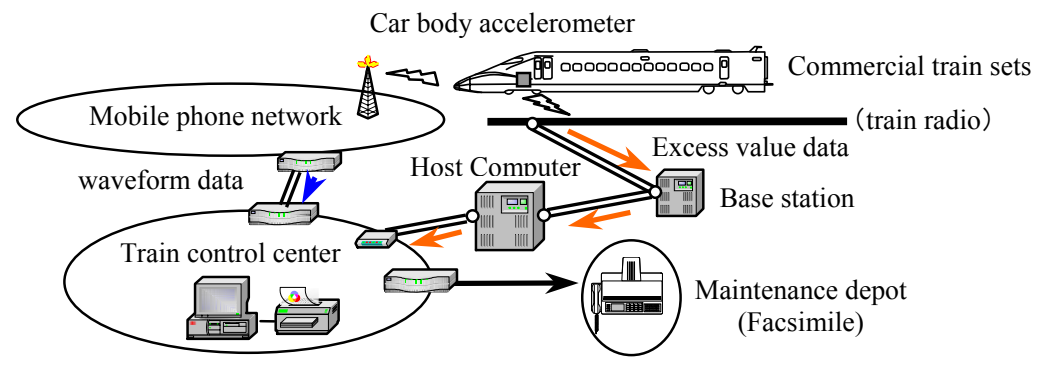

Figure 10: $\quad$ Outline of RAIDARSS 2+.

A key role of this device is to monitor track conditions by measuring car body acceleration in order to ensure safe train operation. However, because the latest N700 series introduced in 2007 is equipped with many devices to improve riding comfort, dampers to reduce vibration, and air suspensions that tilt the car body to run faster on curves, monitoring track conditions by measuring car body acceleration becomes more difficult. Therefore, accelerometers are installed not only on the car body but also on the axle box where vibrations caused by track conditions are directly detected by RAIDARSS on N700 series cars (Fig. 11).

Double integration of the lateral axle-box acceleration does not equal lateral track irregularity because there is a gap between the rail and wheel flange. However, double integration of the vertical axle-box acceleration does equate to vertical track irregularity because wheels are continually in contact with the rails. Because most track irregularities that tend to increase rapidly are mainly vertical track irregularities, it is very beneficial to be able to detect them. Therefore, RAIDARSS-3 for the N700 series has been developed as a digital inertial measuring device. RAIDARSS 3 for commercial trains offers an ideal track monitoring system that is able to precisely measure track irregularities every day. 
Table 1: $\quad$ Main functions of RAIDARSS 2+.

\begin{tabular}{|l|l|}
\hline \multicolumn{1}{|c|}{ Function } & \multicolumn{1}{c|}{ Description } \\
\hline $\begin{array}{l}\text { (1) Reporting excess } \\
\text { values and locations }\end{array}$ & $\begin{array}{l}\text { If the measured accelerations exceed the predetermined target } \\
\text { maintenance values, RAIDARSS automatically reports the } \\
\text { measured values and locations of the irregularities to the train } \\
\text { control center and track maintenance depots. }\end{array}$ \\
\hline $\begin{array}{l}\text { (2) Calculating } \\
\text { distance and speed }\end{array}$ & $\begin{array}{l}\text { RAIDARSS calculates the exact running distance and speed by } \\
\text { counting the number of wheel rotations. }\end{array}$ \\
\hline $\begin{array}{l}\text { (3) Correcting wheel } \\
\text { diameters }\end{array}$ & $\begin{array}{l}\text { RAIDARSS automatically corrects wheel diameters by } \\
\text { calculating running distance and detecting position information } \\
\text { from wayside coils. }\end{array}$ \\
\hline $\begin{array}{l}\text { (4) Sensing opposing } \\
\text { trains }\end{array}$ & RAIDARSS senses opposing trains by optical sensor. \\
\hline $\begin{array}{l}\text { (5) Recording } \\
\text { measurement data }\end{array}$ & RAIDARSS records measurement data for ten runs to a PC card. \\
\hline $\begin{array}{l}\text { (6) Transmitting } \\
\text { waveform data }\end{array}$ & $\begin{array}{l}\text { RAIDARSS transmits waveform data to a terminal on the } \\
\text { ground through a mobile phone network. }\end{array}$ \\
\hline
\end{tabular}

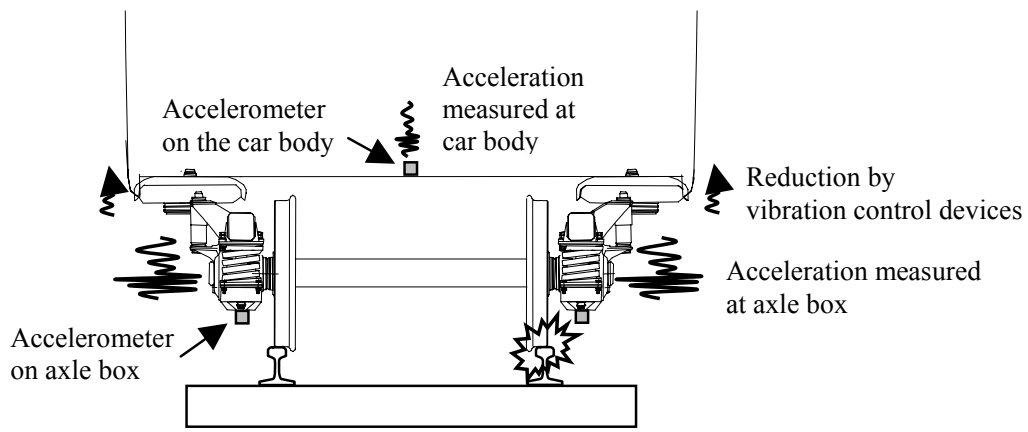

Figure 11: Accelerometer installation points for RAIDARSS on N700 series cars.

\section{Test results of the RAIDARSS 3 prototype}

A RAIDARSS 3 prototype was produced and tested using an axle box acceleration of commercial trains. The two algorithms described in section 3 above were tested using the prototype device. Fig. 12 shows the results: (a) is the result at high speed, and (b) is the result at low speed. For comparison, measurement signals from an existing track geometry car are shown and all data is adjusted wavelength to $6-50 \mathrm{~m}$. At high speed, the waveforms of both algorithms are very similar to the track geometry car. Therefore, this confirms that the digital inertial measurement method achieves sufficient measurement precision. At low speed the waveform from the frequency variable difference filter is more similar to the track geometry car than that of the digital HPF. Because the two different algorithms were tested on different trains, it is 
impossible to conclude that these results are caused solely by the different algorithms. However, it can be confirmed that the algorithm using frequency variable difference filter does achieve sufficient measurement precision even at low speed.

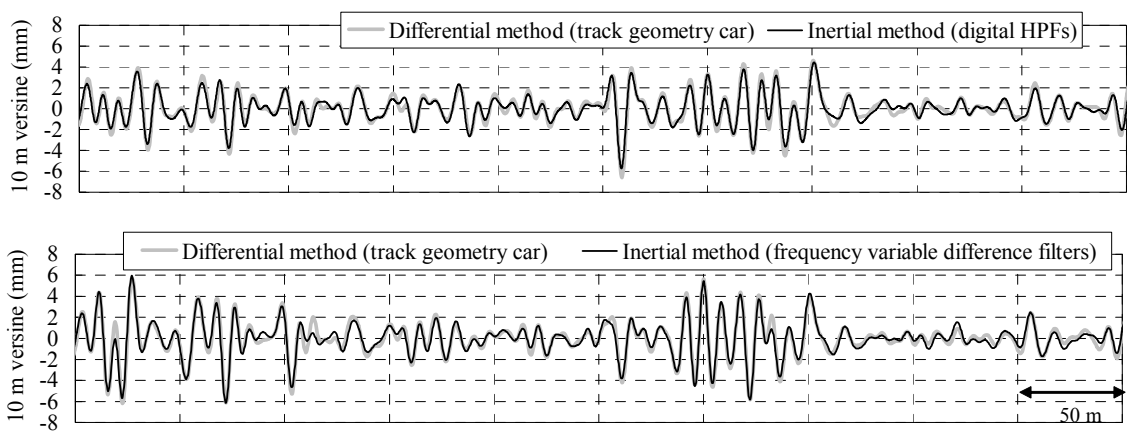

(a) High speed (approx. $260 \mathrm{~km} / \mathrm{h}$ )

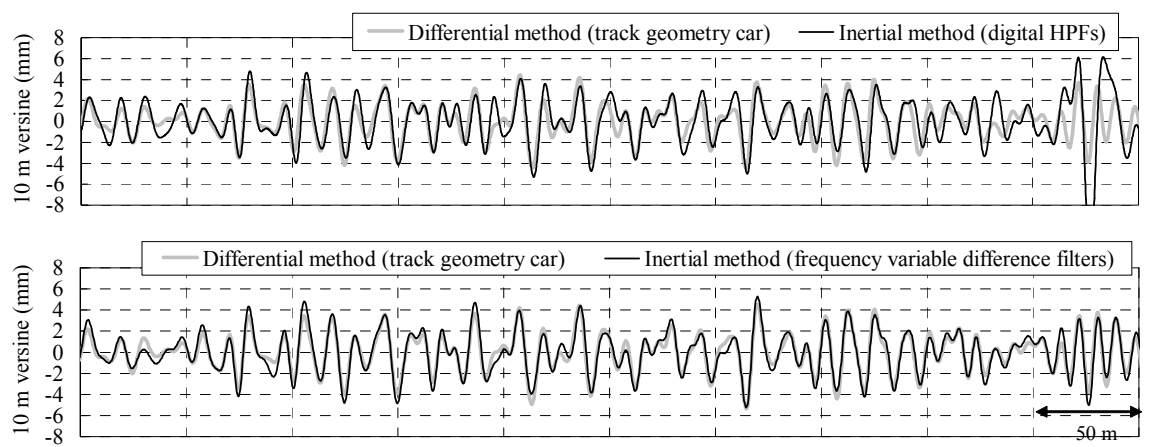

(b) Low speed (approx. $60 \mathrm{~km} / \mathrm{h}$ )

Figure 12: Results of measurement precision of digital inertial measuring device.

\section{Conclusion and future topics}

In this paper, two different digital inertial algorithms for track geometry recording were proposed and test results were shown from a prototype device that applied these algorithms.

1. The algorithm using digital HPFs and the algorithm using frequency variable difference filter that utilized the $10 \mathrm{~m}$ versine measurement property has been proposed for digital inertial measuring devices used for recording track geometry. The algorithm with frequency variable difference filter enables much lighter hardware processing for calculation compared with digital HPFs, and much lower measurable lower limit speed.

2. A prototype device applying the two digital inertial algorithms proposed in this paper was produced and tested on commercial trains. As a result, it was 
confirmed that the measurement precision of the digital inertial algorithms equals the precision of the track geometry car. Especially the algorithm using frequency variable difference filter does achieve sufficient measurement precision even at low speed.

This study confirms that a complete digital inertial measuring device is feasible, paving the way for production of a new track monitoring system that is able to precisely measure track irregularities on commercial trains on a daily basis. The new RAIDARSS 3 track condition monitoring device will be installed on six N700 train sets during fiscal year 2008, with the total system going into test operation in March 2009.

\section{References}

[1] Y. Yamaguchi, K. Kondo, Y. Sato and M. Shirozume. Development of Track State Confirming Machine (TRASC). Proc. of the $5^{\text {th }}$ International Heavy Haul Conference, 1993.

[2] M. Miwa and Y. Sato. Measurement and Analysis of Track Irregularity on Super-High Speed Train. Proc. of the WCRR, 1996.

[3] T. Otake, Y. Naganuma and Y. Sato. Rectification of Distorted Track Irregularity Record Obtained by Inertia Method. Proc. of the $6^{\text {th }}$ International Heavy Haul Conference, 1997.

[4] S. Nakamura, T. Ishikawa and M. Kotani. An Approach of Realizing a Linear-Phase Filter with a Multiple-Notched Property. IEEE Trans., Instrumentation and Measurement, Vol. IM-32, No.4, pp.458-462, 1983.

[5] Y. Naganuma, W. Suzuki, K. Itaya, T. Kohama and S. Nakamura. "A complete digital inertial measurement system for railway tracks", Computers in railway VII (proceedings of COMPRAIL 2000), pp. 82-95, (2000). 